
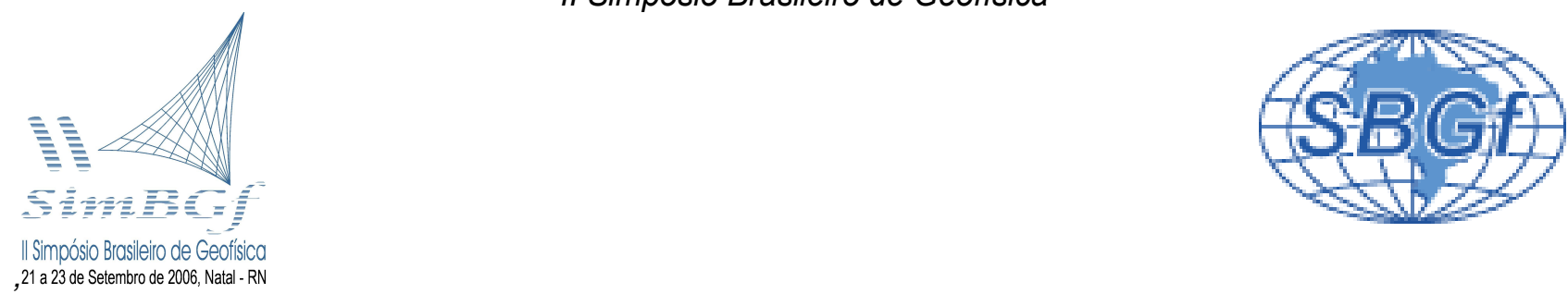

\title{
DETECÇÃO AUTOMÁTICA DAS PRIMEIRAS FASES: APLICAÇÃO EM REFRAÇÃO SÍSMICA PROFUNDA
}

Frederico Xavier de Melo, Departamento de Engenharia Elétrica, Universidade de Brasília, UnB Geovany Araújo Borges, Departamento de Engenharia Elétrica, Universidade de Brasília, UnB José Eduardo Pereira Soares, Instituto de Geociências, Universidade de Brasília, UnB

Copyright 2004, SBGf - Sociedade Brasileira de Geofísica

Este texto foi preparado para a apresentação no I Simpósio de Geofísica da Sociedade Brasileira de Geofísica, São Paulo, 26-28 de setembro de 2004. Seu conteúdo foi revisado pela Comissão Tecno-científica do I SR-SBGf mas não necessariamente representa a opinião da SBGf ou de seus associados. E proibida a reprodução total ou parcial deste material para propósitos comerciais sem prévia autorização da SBGf.

\section{Resumo}

Este trabalho aborda o problema da construção da curva de tempo de trânsito a partir da detecção das primeiras chegadas em registros de refração sísmica profunda. $O$ método empregado na estimação recursiva e continuada com filtro de Kalman em um modelo autoregressivo para o ruído sísmico de fundo. A detecção da quebra de fase é determinada a partir da diferença na distribuição de probabilidade entre o ruído de fundo e a presença do evento sísmico. Este trabalho é parte de um algoritmo que propõe a geração automática de um modelo 1Dmédio da crosta continental a partir dos traços adquiridos provenientes de fontes controladas.

\section{Introdução}

As curvas de tempo de trânsito são obtidas a partir do tempo de chegada das ondas mecânicas provenientes de uma fonte sísmica natural ou controlada. Sua construção dá-se pela combinação do tempo de chegada

$t_{a}$ do evento em um registro localizado a uma distância

$x_{r}$ de uma fonte sísmica. A partir da análise da curva de tempo de trânsito é possível estabelecer paradigmas e modelos acerca de diversas camadas e profundidades abaixo da superfície, inferindo sobre as propriedades físicas e estruturação do meio.

A observação da curva de tempo de trânsito, aliada à teoria de propagação das ondas elásticas, permite obter possíveis soluções para o problema inverso, ou seja, inferir sobre as propriedades físicas do meio a partir dos daddo adquiridos. Em estudos de refração profunda, um dos objetivos principais encontra-se na profundidade e topografia da descontinuidade de Moho. Amplamente conhecido em países do Hemisfério Norte e Austrália, o método ainda não obteve muita significância no Brasil, Das contribuições já realizadas dentro deste âmbito, destacam-se os trabalhos (Perosi, 2000, Berrocal et al., 2004; Soares, 2005, Melo, 2005) e àqueles propostos para a Província Borborema (Fuck et al, 2006).
Este trabalho é parte de pesquisa que propõe um paradigma para geração de modelo 1D-médio da crosta continental a partir da leitura e interpretação automática do tempo de trânsito das primeiras chegadas e da reflexão da Moho com base nos levantamentos de refração sísmica profunda. $\mathrm{O}$ algoritmo divide-se em: i) construção do tempo de trânsito a partir da leitura automática das primeiras chegadas; ii) identificação dos alinhamentos das fases diretas $(\mathrm{Pg})$ e refratada na Moho $(P n)$ e determinação de modelo da crosta com espessura mínima; iii) identificação das reflexões da Moho (PmP) e cálculo do modelo 1D-médio. A primeira etapa foi testada com sucesso em telessísmos (Melo et al., 2005). Este resumo apresenta um algoritmo semelhante aplicado aos dados de refração. A segunda etapa é apresentada e discutida nesse encontro. A terceira etapa envolve a determinação de fases internas relativas às chegadas da ondas refletidas na Moho.

O ponto de partida na determinação de um modelo 1D médio da crosta continental consiste na observação do tempo de chegadas das primeiras quebras ( $\mathrm{Pn}$ e $\mathrm{Pg}$ ) ao longo de sensores espalhados por uma linha de refração sísmica profundo.

O grande volume de dados torna a análise manual dos registros adquiridos um fator limitante em tempo para obtenção das curvas de tempo de trânsito. Diversas técnicas foram desenvolvidas no intuito de automatizar o processo de análise manual do dados. Vale citar metodologias que empregam medições regulares da energia do traço sísmico (Allen, 1982), ou que fazem uso do grau de polarização em sistemas de aquisição triaxial. (Schimmel et al.,2003).

O problema abordado neste resumo é endereçado à construção da curva de tempo de trânsito considerando as fases primárias no traço sísmico. Realiza-se este procedimento a partir da identificação automática do tempo de chegada por meio de técnicas de filtragem estocásticas baseado em um modelo a priori.

Diferentemente da filtragem estocástica de (Halpeny, 1984), o método proposto segue a vertente da identificação em tempo real dos coeficientes do modelo autoregressivo (AR) estabelecidos para o ruído de fundo empregando-se o Filtro de Kalman. Através de um teste probabilístico qui-quadrático baseada na distância de Mahalanobis, o algoritmo proposto rastreia de forma contínua os coeficientes AR para verificar se estão de acordo com o modelo estabelecido inicialmente para o ruído Gaussiano idependente e identicamente distribuído 
(i.i.d.). Caso o teste falhe, o métdodo encara com a provável de um evento. Banco de dados utilizados em estudos de refração sísmica profunda são apresentados para avaliação do método proposto.

\section{Metodologia/ Problema Investigado}

Filtro de Kalman e modelo de identificação das primeiras quebras

Em um sismograma composto por uma componente de registro $z[t]$, representado como uma série temporal discreta $z_{n}=\left[z_{1}, \ldots, z_{n}\right]$, a construção de um modelo autoregressivo (AR) de ordem $m$ fornece uma simples descrição sobre suas características em diferentes janelas de tempo. O modelo consiste na representação da amostra presente a partir da combinação linear de $m$ amostras passadas acrescidas por um resíduo e[t]. Assume-se $\epsilon[t]$ como um parâmetro de incerteza caracterizado como uma seqüência i.i.d. Gaussiana com média $E\{\epsilon[t]\}=0$ e variância $E\left\{(\epsilon[t])^{2}\right\}=\sigma^{2}$.

De forma a preservar as características estatísticas de $\epsilon[t]$, a ordem $\mathrm{m}$ do modelo AR deve ser dimensionada de acordo com a complexidade da série temporal a ser representada. Um exemplo sobre a diferença dos comportamentos dos resíduos é mostrada na Figura 1, onde $\epsilon[t]$ possui distribuições distintas para as janelas compostas pelo ruído de fundo e pelo mesmo ruido acrescido do sinal sísmico.
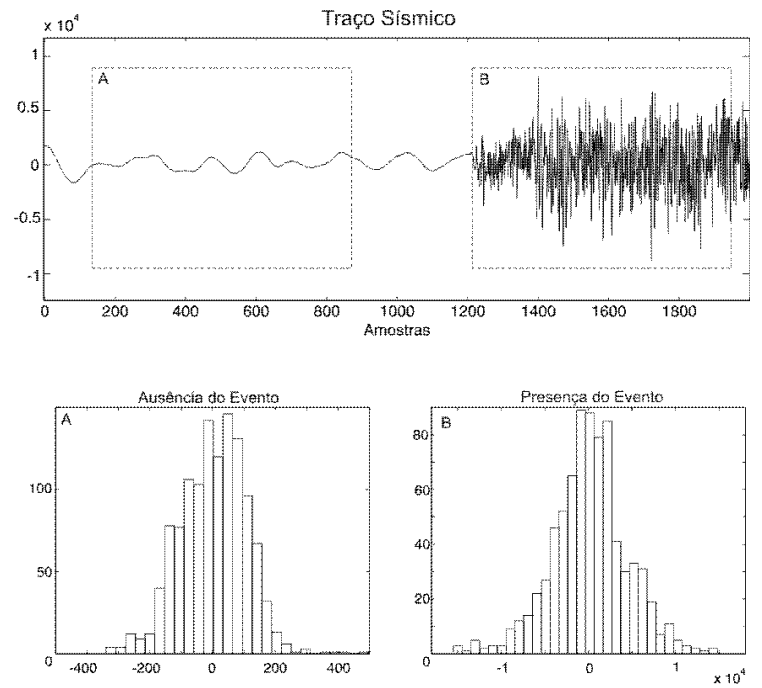

Figura 1 -Resíduos do modelo AR de quarta ordem para região do traço em $A$. A janela em (1) mostra o histograma para o ruído de fundo (B) e (2) com a presença de um evento (C).

As diferentes características estatísticas entre os resíduos do modelo AR permitem estabelecer técnicas para observação e rastreamento de intervalos, dentro do traço sísmico, os quais a gaussianidade seja preservada. A partir da utilização do Filtro de Kalman, este trabalho estabelece um modelo de estimação recursiva para o processo de observação do ruído colorido. Este filtro estocástico consiste em um estimador ótimo em um senso de mínima média quadrática do erro de estimação para o seguinte modelo linear de estado e observação (Jazwinski, 1970)

$$
\begin{aligned}
& \text { Equação de estados } \boldsymbol{x}[k]=\boldsymbol{A}[k] \boldsymbol{x}[k-1]+\boldsymbol{w}[k] \\
& \text { Equação de observação } z[k]=\boldsymbol{H}[k] \boldsymbol{x}[k]+v[k],
\end{aligned}
$$

onde $\boldsymbol{x}[k]$ é o vetor de estados $(n, 1), \boldsymbol{A}[k]$ é a matriz de transiçao de estados $(n, n), \boldsymbol{w}[k]$ é uma seqüência $(n, 1)$, de ruído branco Gaussiano com média zero, $\boldsymbol{z}[k]$ é o traço sísmico representado como vetor de observação escalar, $\boldsymbol{H}[k]$ é a matriz de observação

$(1, n)$ e $\boldsymbol{v}[k]$ é uma seqüência escalar de ruído branco Gaussiano. As seqüências Gaussiana são decorrelacionadas e que

$$
\begin{aligned}
& E\left\{\boldsymbol{w}[k] \boldsymbol{w}[i]^{T}\right\}=\boldsymbol{Q}[k] \text {, se } k=i, \text { ou } 0 \text {, caso contrário } \\
& E\left\{v[k] v[i]^{T}\right\}=R[k] \text {, se } k=i \text {, ou } 0 \text {, caso contrário }
\end{aligned}
$$

onde $\boldsymbol{Q}[k]$ é uma matriz quadrática com dimensão $(n, n)$ e $R[k]$ um vetor escalar $(n, 1)$.

O problema básico do filtro consiste em obter estimativas $\hat{\boldsymbol{x}}[k \mid k]$ de $\boldsymbol{x}[k]$ a partir da medições da medição $z[k]=[z[k], \ldots, z[k]]$, de forma a minimizar, empregando técnicas de estimação recursiva, o erro médio quadrático dado pelo traço da matriz de covariância do erro a posteriori

$$
P[k \mid k]=E\left\{(\boldsymbol{x}[k]-\hat{\boldsymbol{x}}[k \mid k])(\boldsymbol{x}[k]-\hat{\boldsymbol{x}}[k \mid k])^{T}\right\} .
$$

O vetor de estados proposto para observar a evolução dos coeficientes AR, dentro do modelo proposto para representação do sismograma é apresentado da seguinte forma

$$
\boldsymbol{x}[k]=\left[\begin{array}{llll}
a_{1}[k] & a_{2}[k] & \ldots & a_{m}[k]
\end{array}\right]^{T},
$$

onde $a_{1}[k], a_{2}[k], \ldots, a_{m}[k]$ são os coeficientes do modelo AR de ordem $m$. Sua evolução temporal é descrita através de um sistema de primeira ordem dado por

$$
\begin{gathered}
a_{1}[k]=a_{1}[k-1]+T_{a} \dot{a}_{1}[k-1]+w_{1}[k], \\
a_{2}[k]=a_{2}[k-1]+T_{a} \dot{a}_{2}[k-1]+w_{2}[k], \\
\vdots \\
a_{m}[k]=a_{m}[k-1]+T_{a} \dot{a}_{m}[k-1]+w_{m}[k],
\end{gathered}
$$

onde $T_{a}$ é o período de amostragem da aquisição da série temporal e $w_{i}[k]$ é o erro associado ao modelo de evolução do i-ésimo coeficiente. $\dot{a}_{i}[k-1]$ representa a taxa de variação do sistema de evolução dada da seguinte forma

$$
\begin{gathered}
\dot{a_{1}}[k]=\dot{a}_{1}[k-1]+w_{m+1}[k], \\
\dot{a_{2}}[k]=\dot{a}_{2}[k-1]+w_{m+2}[k], \\
\vdots \\
\dot{a}_{m}[k]=\dot{a}_{m}[k-1]+w_{2 m}[k] .
\end{gathered}
$$

A identificação do instante de chegada do evento sísmico baseia-se no emprego de um teste estatístico que, aliado à utilização da filtragem estocástica, verifica se as propriedades estatísticas dos resíduos dos coeficientes do modelo AR são seqüências Gaussianas. Caso o teste falhe aponte uma falha, assume-se que a amostra não pertence à distribuição dos resíduos passados, o que corresponde à chegada da fase primária do evento. 


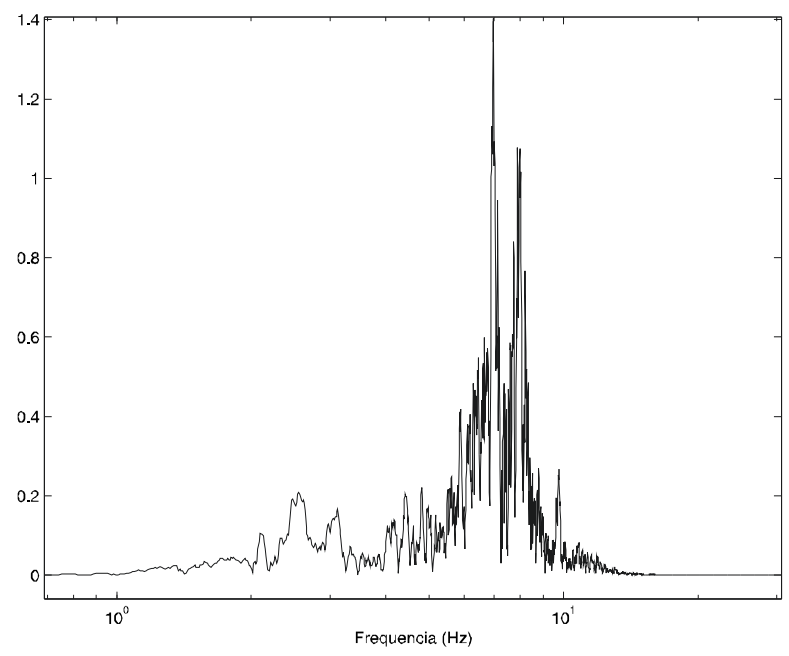

Figura 2 - Espectro de freqüência de um traço sísmico de refração profunda. Nota-se a maior concentração de energia entre 2 e $16 \mathrm{~Hz}$.

A hipótese probabilística adotada para verificação dos resíduos é mesurada através do teste qui-quadrático através da distância da Mahalanobis (Duda et al.,2000) e indica o quanto o resíduo da amostra atual do registro é compatível com o modelo de evolução estipulado para os resíduos do ruído de fundo. Para um variável aleatória $e \simeq N\left(0, \sigma_{e}^{2}\right)$, uma amostra tem $95 \%$ de chance de pertencer à distribuição de $e$ se

$$
e[k]^{2} / \sigma_{e}^{2}<d,
$$

onde $d=3.84$ e $e[k]$ representa o termo de inovação do filtro de Kalman, ou seja, a diferença entre a amostra atual $z[k]$ e o modelo estipulado a priori. Como dito anteriormente, a não aprovação no teste significa uma incompatibilidade entre o resíduo e modelo de evolução definido a priori. Esse fator indica que houve a chegada de um evento sísmico e o processo de estimação é interrompido.

Mais detalhes sobre o funcionamento do filtro de Kalman e do algoritmo de detecção a partir do modelo de evolução dos resíduos do modelo AR de ordem $m$ encontram-se em (Jazwinski, 1970), (Brown et al., 1997) e (Melo et al, 2006).

\section{Pré-processamento dos dados}

Em estudos que envolvem levantamentos de refração sísmica profunda, os dados são compostos a partir de uma fonte sísmica controlada e pelo registro em sensores espalhados ao longo de uma linha sísmica. O comprimento da linha é determinado de acordo com o objeto de interesse do estudo, bem como o número de equipamentos disponíveis para sua realização.

O problema abordado neste trabalho concentra-se na construção do tempo de trânsito das ondas sísmicas a partir da identificação das primeiras chegadas dos eventos provenientes de uma fonte sísmica controlada.

A Figura 2 mostra o espectro de freqüência de um registro localizado à $50 \mathrm{~km}$ de uma fonte controlada utilizada para levantamento de refração sísmica. Nota-se que as componentes do espectro concentram-se, em sua maioria, na região de 2 a $16 \mathrm{~Hz}$. Baseado na constatação, estabeleceu-se que os traços adquiridos seriam filtrados para realçar somente os espectro de interesse. Para tanto, utilizou-se filtros passa-banda do tipo Butterworth de terceira ordem com freqüências de corte entre 2 e $4 \mathrm{~Hz}$. Além da aplicação do filtro no domínio da freqüências, todos os traços utilizados foram normalizados no inuito de padronizar os valores dos parâmetros de ajuste do algoritmo de detecção.

\section{Parâmetros de ajuste e otimização do algoritmo}

O filtro de Kalman estabelece, como determinação a priori (Brown et al., 1997), o completo conhecimento das características estatísticas das ruídos associadas ao modelo de medição $(\boldsymbol{Q}[k])$ e de observação $(R[k])$. Contudo, esse quesito não é verificado em muitas aplicações as quais as incertezas não podem ser determinadas de maneira concisa, levando, em alguns casos, à divergência da estimação recursiva do filtro.

Este trabalho assume que as incertezas $\boldsymbol{Q}$ e $R$ possuem um valor conhecido a priori e invariante no tempo. Estes são os parâmetros livres do algoritmo de detecção, devendo ser validados através da antiga prática associada à arte de ajuste do filtro de Kaman.

Devido ao posicionamento dos sensores em linha, o tempo de chegada do evento está estritamente relacionado à distância que o equipamento encontra-se da fonte sísmica. A partir desta constatação, o algoritmo de detecção realiza a busca pelo a chegada do evento dentro de um intervalo determinado pelo usuário. Com isso, o tempo de processamento dos traços é reduzido consideravelmente.

\section{Pós-processamento dos dados}

De modo a previnir falsos positivos na determinação do tempo de chegada no traço sísmico, rotinas de processamento nos dados são adotadas de forma a evitar as diversas variações que podem ocorrer dentro da janela de analise.

Variações de baixa freqüência não inferem no resultado do algoritmo proposto, pois o procedimento continuado de estimação do modelo AR permite sua adaptação à mudanças de baixa freqüência no registro do traço sísmico. Variação de temperatura no ambiente de operação equipamento constitui um exemplo de possível interferência de baixa freqüência.

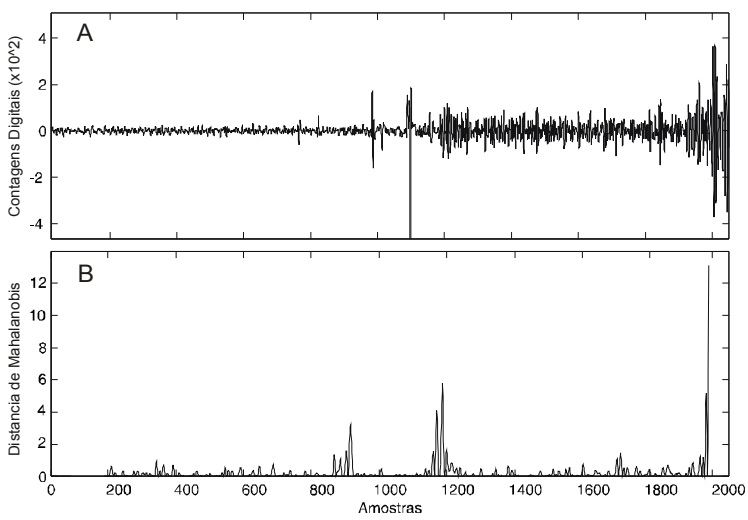

Figura 3 - Variações de alta freqüência (spikes). 


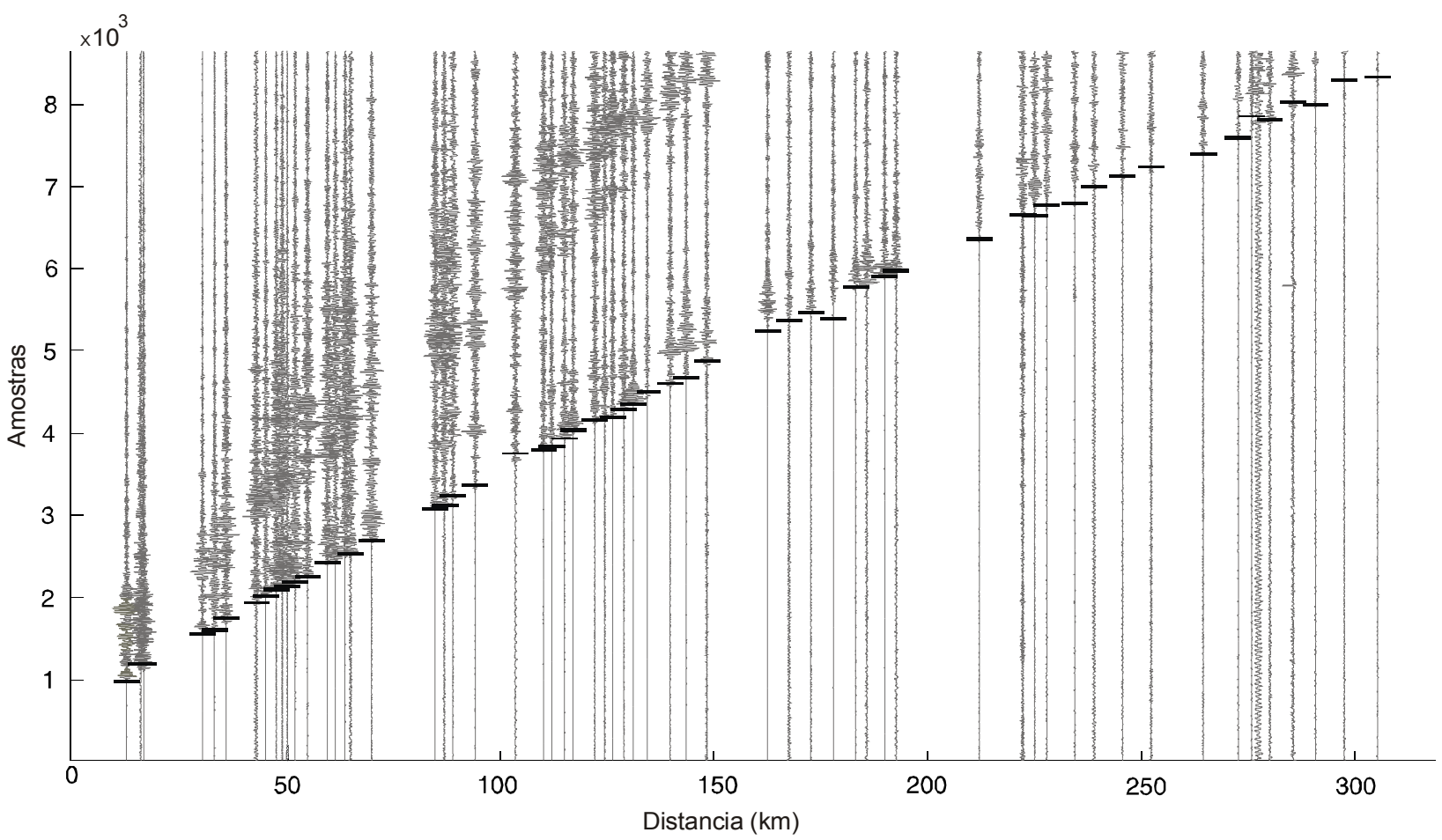

Figura 4 - Resultado do algoritmo de detecção das primeiras chegadas para o tiro 1 de Cavalcante.

Para variações de alta freqüência dentro de um curto intervalo de tempo (spikes) é necessária a implementação de uma rotina que evite a interpretação errônea do tempo de chegada pelo algoritmo.

A característica de um spike é que, dentro da janela de ruído de fundo, os resíduos do modelo AR estmado sejam maiores do que o limiar estipulado para o teste de verificação da distância de Mahalanobis. No entanto, o resíduo não atinge valores muito elevados devido à curta duração de tempo durante a ocorrência do spike.

Partindo dessa premissa, o algoritmo trabalha com uma faixa de segurança de detecção com $10 \%$ acima do valor do limiar estabelecido na equação (8) .Caso o algoritmo identifique uma possível chegada de evento sísmico, uma nova verificação da hipótese probabilística é realizada com o valor de $d=3.84$. Com isso, espera-se que o algoritmo seja mais robusto à ocorrência de spikes e qualquer tipo de variação de alta freqüência dentro do traço sísmico. A Figura 3 mostra o comportamento na amplitude dos resíduos quando ocorre a presença de spikes antes da chegada do evento sísmico.

\section{Resultados e Discussões}

Dados reais foram utilizados na aplicação do método de construção do tempo de trânsito provenientes da identificação das primeiras quebras dos traços sísmicos. Os dados empregados consistem nos registros dos tiros 1 e 7 da linha de refração sísmica profunda de Cavalcante (Soares et al., 2005). Esta linha foi levantada com 120 sensores distribuídos ao longo de, aproximadamente, $300 \mathrm{~km}$ de extensão. A taxa de amostragem para os traços foi de $166 \mathrm{~Hz}$.

Para as duas bases de dados utilizadas, o valor do vetor de estado $\boldsymbol{x}[0]$ foi determinado pelo cálculo dos coeficientes AR de ordem $m$ empregando o método recursivo de Levinson para solução das equações de Yule-Walker (Pollock, 1999). Para cada traço analisado, 100 amostras iniciais (composta somente por ruído sísmico de fundo) foram selecionadas para determinação dos coeficientes.

A partir da descorrelação entre os coeficientes iniciais, definiu-se que o valor da matriz de covariância $\boldsymbol{P}[0]$ teria forma diagonal e que se valor inicial deveria ser sudficiente para evitar a divergência do filtro no início do processo de estimação.

Através de prévia inspeção visual, 58 registros do tiro 1 e 72 registros do tiro 7 foram descartados por não apresentarem qualquer tipo de sinal presente. $O$ alto índice de descarte está relacionado às condições de funcionamento do equipamento e/ou ao elevado nível de ruído de fundo, capaz de mascarar o registro dos tiros durante a ocorrência do evento controlado.

Um modelo AR de terceira ordem foi escolhido para representar a evolução do ruído de fundo nos registros do tiro 1. Os parâmetros de ajuste adotados foram $R=5,00 \cdot 10^{-6}$ e a matriz diagonal $\boldsymbol{Q}$ de valores $q_{i j}=8.00 \cdot 10^{-7}, i \leq 4$ e $q_{i j}=1.00 \cdot 10^{-8}, 5 \leq i \leq 8$. A Tabela 1 mostra o resultado comparativo para 15 traços selecionados ao longo da linha de refração sísmica. Os resultados são expostos em conjunto com a determinação do tempo de chegada por uma analista de dados. Os traços dos dados utilizados, bem como a determinação dos tempos de chegada, estão expostos na Figura 4. 


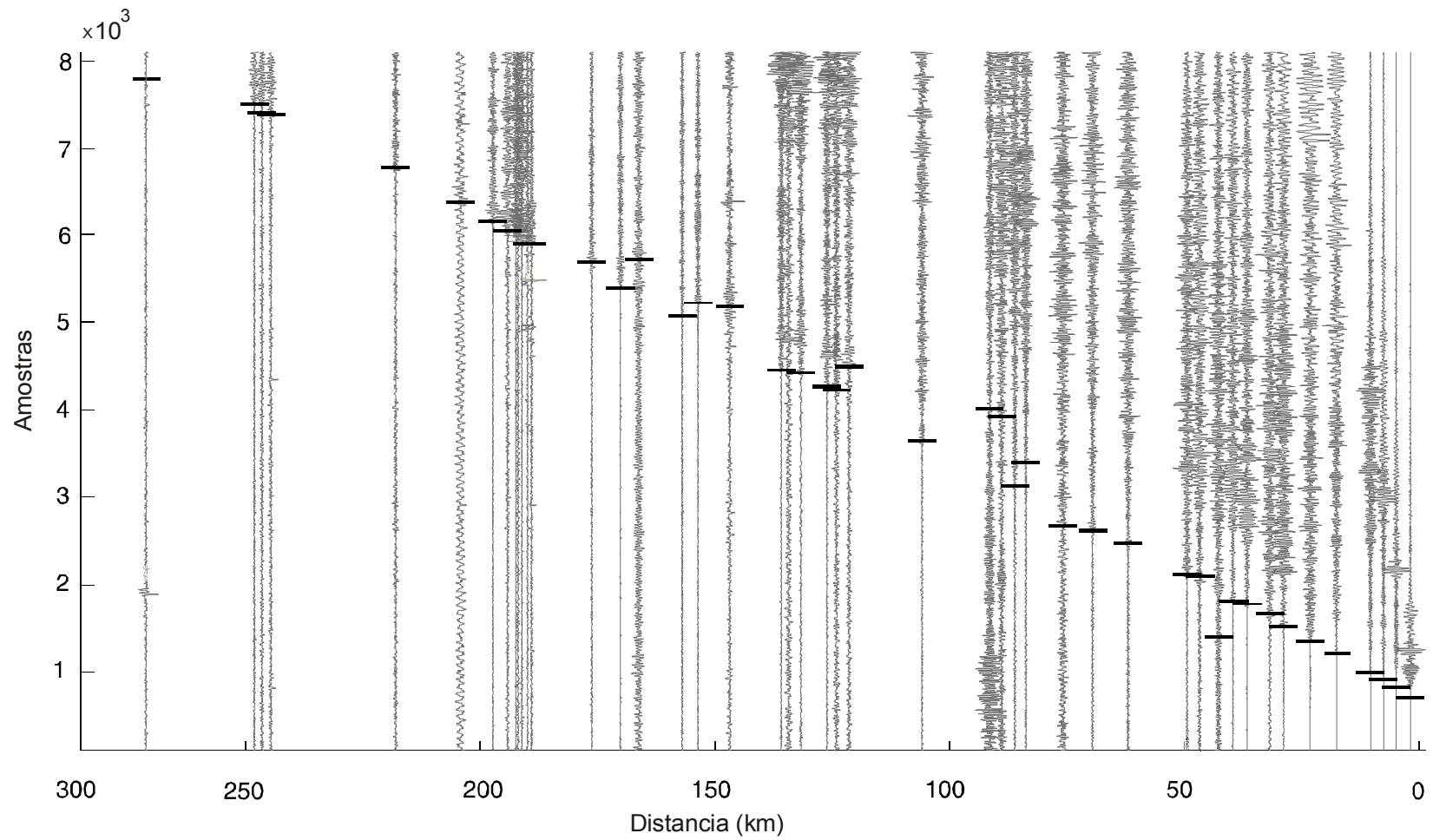

Figura 5 - Resultado do algoritmo de deteç̧ão das primeiras chegadas para o tiro 7 de Cavalcante.

O modelo AR definido para o tiro 7 é de segunda ordem e os parâmetros de ajuste adotados foram $R=8,00 \cdot 10^{-5}$ a matriz $Q$ com valores $q_{i j}=8.00 \cdot 10^{-7}, i \leq 4$ e $q_{i j}=1.00 \cdot 10^{-9}, 5 \leq i \leq 8$. A Tabela 2 mostra o resultado comparativo do algoritmo para os traços selecionados. A Figura 5 mostra a determinação gráfica do método.

Para ambos os tiros considerados, o algoritmo mostrouse eficiente na detecção da primeira quebra mesmo em traços que apresentavam uma baixa relação sinal/ruído. Os dados apresentados nas tabelas 1 e 2 mostram que, em $78 \%$ dos traços selecionados, a diferença entre a determinação da chegada do método proposto e do analista humano foi menor do que 20 amostras (aproximadamente $120 \mathrm{~ms}$ a uma taxa de amostragem de $166 \mathrm{~Hz})$.

Tabela 1 - Determinação de tempo de chegada - tiro 1

\begin{tabular}{|c|c|c|}
\hline Distância da fonte $\mathbf{( k m )}$ & Analista & Algoritmo \\
\hline 12.65 & 990 & 975 \\
\hline 47.66 & 2060 & 2096 \\
\hline 84.83 & 3060 & 3060 \\
\hline 103.57 & 3650 & 3744 \\
\hline 148.36 & 4840 & 4875 \\
\hline 225.04 & 6600 & 6650 \\
\hline 264.43 & 7390 & 7400 \\
\hline 305.28 & 8300 & 8324 \\
\hline
\end{tabular}

Para o tiro 1, o evento de registrado por uma estação localizada a $264.43 \mathrm{~km}$ da fonte sísmica controlada, a determinação do tempo de chegada não apresentou diferença significativa entre 0 analista e o método proposto. Nota-se que, mesmo com a baixa relação sinal/ruído do traço, o limiar determinado para o teste probabilístico pela distância de Mahalanobis é atingido somente na região onde há a ocorrência do evento sísmico. Neste caso, a diferença entre as chegadas determinadas de forma automática e manual diferem-se de 10 amostras (60ms adquiridos a $166 \mathrm{~Hz}$ ).

A baixa qualidade dos registros localizados a 83.76 e $124.12 \mathrm{~km}$ da fonte do tiro 7, impossibilitou a marcação de chegada de fase pelo analista humano. No entanto, o algoritmo apontou um provável tempo de chegada baseado na observação dos resíduos dos coeficientes do modelo AR. A correta marcação foi constatada pela marcação realizada nos traços adjancentes, os quais apontavam coerência na construção do tempo de trânsito no levantamento da linha de refração sísmica.

Houveram registros onde 0 método automático determinou a chegada do evento em um tempo posterior à sua ocorrência. Isto deve-se ao grau de complexidade da série temporal na região entre o ruído de fundo e a quebra do evento sísmico. No tiro 7, por exemplo, o registro localizado a $134.32 \mathrm{~km}$ da fonte, a distância de Mahalanobis calculada atinge o limiar de 3.84 somente na amostra 5000 (300 amostras depois da quebra da fase). Nas amostras anteriores, a distância calculada não chega a $5 \%$ do limiar estabelecido.

Para ambos os tiros utilizados, 24 eventos submetidos ao algoritmo deste trabalho apresentaram atrasos na determinação da fase $\mathrm{Pn}$ e $\mathrm{Pg}$ maiores que 50 amostras. Verificou-se que a característica predominante para estes registros era a baixa relação sinal/ruído. Eventos 
que apresentaram um alto grau de sobreposição do ruído sobre a real chegada da primeira quebra, o método de estimação consegue identificar somente a sua ocorrência, ou seja, o algoritmo determina a chegada após sua ocorrência.

Tabela 2 - Determinação do tempo de chegada - tiro 7

\begin{tabular}{|c|c|c|}
\hline Distância da fonte (km) & Analista & Algoritmo \\
\hline 1.86 & 706 & 706 \\
\hline 75.89 & 2605 & 2620 \\
\hline 83.76 & - & 2671 \\
\hline 124.12 & - & 4216 \\
\hline 126.12 & 4276 & 4276 \\
\hline 248.13 & 7501 & 7510 \\
\hline
\end{tabular}

\section{Conclusões}

Este trabalho propôs uma metodologia para construção da curva de tempo de trânsito a partir da identificação das primeiras chegadas nos registros efetuados em levantamentos de refração sísmica profunda para o estudo da crosta continental.

A determinação do tempo de chegada do evento sísmico baseia-se na estimação recursiva dos coeficientes AR de modo a identificar um modelo do processo do ruído de fundo utilizando filtro de Kalman. O modelo de identificação considera a evolução de primeira ordem dos coeficientes, juntamente com a aplicação de um teste probabilístico qui-quadrático baseado na distância de Mahalanobis. Incertezas sobre os modelos de estimação e medição, bem como a ordem do modelo AR para o ruído colorido de fundo, constituem parâmetros livres do algoritmo e devem ser estipulados a priori.

Este trabalho consiste em uma etapa inicial na pesquisa de um método automático para geração do modelo 1D da crosta continental. A automatização das tarefas de detecção das primeiras quebras proporcionam a diminuição o tempo gasto na análise manual dos dados, dado seu grande volume quando empregados em estudos de refração sísmica profunda.

\section{Referências}

ALLEN, R.-1982-Automatic Phase Pickers: Their Present Use and Future Prospects. Bulletin of the Seismological Society of America, 72(6), pp. 225-242

BERROCAL, J., MARANGONI, Y., CÔGO DE SÁ, N., FUCK, R.A., SOARES, J.E.P., DANTAS, E., PEROSI, F., \& FERNANDES, C., Deep seismic refraction and gravity crustal model and tectonic deformation in Tocantins Province, Central Brazil. Tectonophysics, 388, 187-199, 2004.

BROWN, R.G. \& HWANG, P.Y.C.-1997-Introduction to Random Signals and Applied Kalman Filtering, 3rd. ed., John Wiley \& Sons, New York, USA.
FUCK, R.A., BERROCAL, J., ASSUMPÇÃO, M. e outros2006- Estudos geofísicos e tectônicos na Província Borborema. Projeto pesquisa financiado pelo CNPq, rubrica Instituto do Milênio.

HALPENY, J.-1984- A Method of Editing Time Series Observations, Geophysics, 499(5), pp. 521-524.

JAZWINSKI, A.H.-1970- Stochastic Processes and Filtering Theory, 1st ed., Academic Press

MELO, F.X. \& BORGES, G.A. -2005- P-Wave Onset Time Determination Based On Background Noise Autoregressive Model Tracking with Kalman Filtering, In: IASPEI General Assembly, Santiago, Chile.

MELO, F.X. BORGES, G.A \& SOARES, J.E.P.-2006Identificação do Tempo de Quebra de Onda Sísmica P Usando Filtro de Kalman, Submetido para XVI Congresso Brasileiro de Automática, Salvador, Bahia, Brasil.

MELO, S.S.V.-2005- Razão de Poisson da crosta superior da região de Porangatu, Goiás, Província Tocantins: um estudo por refração sísmica. Universidade de Brasília, Instituto de Geociências, Brasília. Tese de mestrado 203.

PEROSI, F.-2000- Refração sísmica profunda no setor sudeste da Província Tocantins. Dissertação de mestrado, IAG-USP. São Paulo.

POLLOCK, D.S.G.-1999- A Handbook of Time Series Analysis, Signal Processing and Dynamics, Vol.1 of Signal Processing and its Applications, 1st ed., Academic Press.

RICHARD, O. DUDA, P.E.H. \& STORK, D.G.-2000Pattern Classification, 2nd. ed., John Wiley \& Sons, New York, USA.

SCHIMMEL, M. \& GALLART, J.-2003- The use of instantaneous polarization attributes for seismic signal detection and image enhancement, Bulletin of the Seismological Society of America, 155, pp. 653-668.

SOARES, J.E.P-2005- Estudos de refração sísmica profunda, função do receptor e gravimetria na Província Tocantins, Brasil central. Universidade de Brasília, Instituto de Geociências, Brasília. Tese de doutorado 73. 